\title{
Evolution of Biological Bandages as First Cover for Burn Patients
}

\author{
Philippe Abdel-Sayed, ${ }^{1,2, *}$ Nathalie Hirt-Burri, ${ }^{1,2}$ \\ Anthony de Buys Roessingh, ${ }^{3}$ Wassim Raffoul, ${ }^{2}$ \\ and Lee Ann Applegate ${ }^{1,2, *}$ \\ ${ }^{1}$ Regenerative Therapy Unit (UTR), Department of Musculoskeletal Medicine DAL, \\ Lausanne University Hospital, Lausanne, Switzerland. \\ ${ }^{2}$ Service of Plastic, Reconstructive \& Hand Surgery, Lausanne University Hospital, Lausanne, Switzerland. \\ ${ }^{3}$ Service of Pediatric Surgery, Lausanne University Hospital, Lausanne, Switzerland.
}

Significance: Cutaneous wound regeneration is vital to keep skin functions and for large wounds, to maintain human survival. In a deep burn, the ability of the skin to heal is compromised due to the damage of vasculature and resident cells, hindering a coordinated response in the regeneration process. Temporal skin substitutes used as first cover can play a major role in skin regeneration as they allow a rapid wound covering that, in turn, can significantly reduce infection risk, rate of secondary corrective surgeries, and indirectly hospitalization time and costs.

Recent Advances: Skin was one of the first tissues to be bioengineered providing thus a skin equivalent; however, what is the current status subsequent to 40 years of tissue engineering? We review the classic paradigms of biological skin substitutes used as first cover and evaluate recent discoveries and clinical approaches adapted for burn injuries cover, with an emphasis on innovative cellbased approaches.

Critical Issues: Cell-based first covers offer promising perspectives as they can have an active function in wound healing, such as faster healing minimizing scar formation and prepared wound bed for subsequent grafting. However, cell-based therapies encounter some limitations due to regulatory hurdles, as they are considered as "Advanced Therapy Medicinal Products," which imposes the same industry-destined good manufacturing practices as for pharmaceutical products and biological drug development.

Future Directions: Further improvements in clinical outcome can be expected principally with the use of cell-based therapies; however, hospital exemptions are necessary to assure accessibility to the patient and safety without hindering advances in therapies.

Keywords: skin graft, temporary substitute, biological bandages, first cover

\section{SCOPE AND SIGNIFICANCE}

TEMPORAL SKIN SUBSTITUTES used as first cover can play a major role in skin regeneration, especially for deep burns, as they allow a rapid wound covering that, in turn, can significantly improve subsequent clinical outcomes. In this review, we discuss the evolution of biological skin substitutes to include innovative cell-

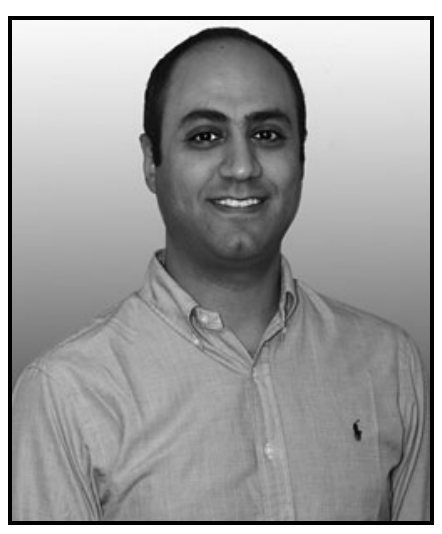

Philippe Abdel-Sayed, PhD

Submitted for publication May 28, 2019. Accepted in revised form July 12, 2019.

*Correspondence: Regenerative Therapy Unit (UTR), Department of Musculoskeletal Medicine DAL, Lausanne University Hospital, EPCR/Croisettes 22, Epalinges, Lausanne 1066, Switzerland (e-mail: philippe.abdel-sayed@chuv.ch).

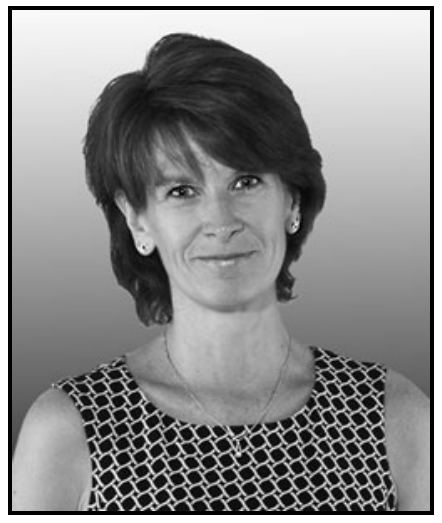

Lee Ann Applegate, PhD

${ }^{*}$ Correspondence: Regenerative Therapy Unit (UTR), Department of Musculoskeletal Medicine DAL, Lausanne University Hospital, EPCR/Croisettes 22, Epalinges, Lausanne 1066, Switzerland (e-mail: lee.laurent-applegate@chuv.ch). 
based therapies, with associated advantages and limitations.

\section{TRANSLATIONAL RELEVANCE}

In a deep burn, the ability of the skin to heal is compromised due to the damage of vasculature and resident cells, hindering a coordinated response in the healing process. The introduction of next generation of biological skin substitutes could stimulate regeneration by the release of a well-proportioned combination of growth factors.

\section{CLINICAL RELEVANCE}

The care of large burn wounds is associated with prolonged hospitalizations and significant costs without mentioning the severe pain, emotional, and physical strain that the burn trauma inflicts to patients. Unmet needs still remain regarding sufficiently advanced wound care therapies to improve healing. Cell-based first covers offer innovative approaches to treat burn wounds and cope with the various burden.

\section{BACKGROUND}

The skin is the largest organ of the human body, having the functions of controlling the body temperature and peripheral fluid balance, allowing sensation (e.g., heat, cold, touch, and pain) and providing a semipermeable protective layer against pathogens. ${ }^{1,2}$ Although the skin has a spontaneous self-regeneration ability, in some cases physiopathological conditions hinder this ability: in chronic wounds, such as in diabetic foot ulcer or pressure ulcer, tissue homeostasis is disrupted because of an altered paracrine signaling between keratinocytes and fibroblasts ${ }^{3}$; in acute wounds (e.g., deep burns), vasculature is damaged or partially damaged, thus limiting the number of cells and factors brought for regeneration. ${ }^{4}$ The time to wound closure is correlated with higher risk of complications such as infection, pain, and scarring occurrence. ${ }^{5}$

Several approaches have been developed over the years to treat cutaneous wounds that have lost their ability of spontaneous healing; split-thickness skin autografting remains the gold standard treatment for covering of large acute and chronic wounds (Fig. 1). ${ }^{6}$ However, immediate wound covering by surgery is not always possible such as in large wounds of severely burned patients, as available surface for donor skin is not sufficient for autografting. In such cases, alternative cell-based strategies have been proven to be life saving, namely by the cultured epithelial autografting (CEA), where the patient's own keratinocytes are expanded to form stratified cell sheets that can be applied topically to the patient for wound closure. ${ }^{7}$

In the mid-90s, we have also implemented at our hospital a variant cell therapy, namely cultured dermal-epidermal autograft (CDEA), which is based on the same principle of stratified cell sheets as for CEA, but allows to recreate a thicker skin graft, because of a double layer composed of autologous fibroblasts and keratinocytes. ${ }^{8}$ CDEA provides better clinical outcome with a skin graft having more functional mechanical properties than a CEA. Nevertheless, CEA and CDEA techniques require a minimum of 3 and 6 weeks, respectively, to expand cells and build bioengineered thin skin grafts, leaving the patient without cutaneous covering for considerable time periods.

Temporary skin substitutes have a crucial function in this specific case, as they are applied on patients during the cell culture phase to prevent fluid loss and decrease pain by nerve covering, but most importantly provide a physical barrier that can reduce infection risks. ${ }^{9}$ Indeed, wound infection is thus the major cause of mortality, morbidity, and wound healing delay. ${ }^{10,11}$ With $>11$ million people affected annually by burn injuries worldwide, ${ }^{12}$ the prevalence of infection in burn units is $\sim 66 \%$. $^{13}$ Temporary skin substitutes may considerably reduce the costs and burden related to prolonged hospitalization by reducing the risk of infection and thus have a major impact on the complications requiring longer hospitalization periods.

In this study, we aim to present the different types of temporary skin substitutes used as first cover for skin burns, with a major emphasis on our own experience with the history of the different first covers used at the burn unit of our hospital, including a comparison of the performance, costs, and regulatory pathway of each temporary cover.

\section{EVOLUTION OF TEMPORARY SKIN SUBSTITUTES IN CLINICAL USE}

Skin substitutes can be classified into two categories: permanent substitutes and temporary substitutes, which we name "first covers" (Fig. 2). Over time, permanent skin substitutes have encompassed all the cell-based grafting approaches, including autografting of cultured and stratified cells with or without the support of regenerative matrices. Temporary skin substitutes have included xenogeneic decellularized skin, allogenic cadaveric human skin, acting mainly as a barrier to external contamination and protection of underlying tissue, but have evolved to more sophisticated bioengineered formulations, allowing rapid would healing. In this section, we will 


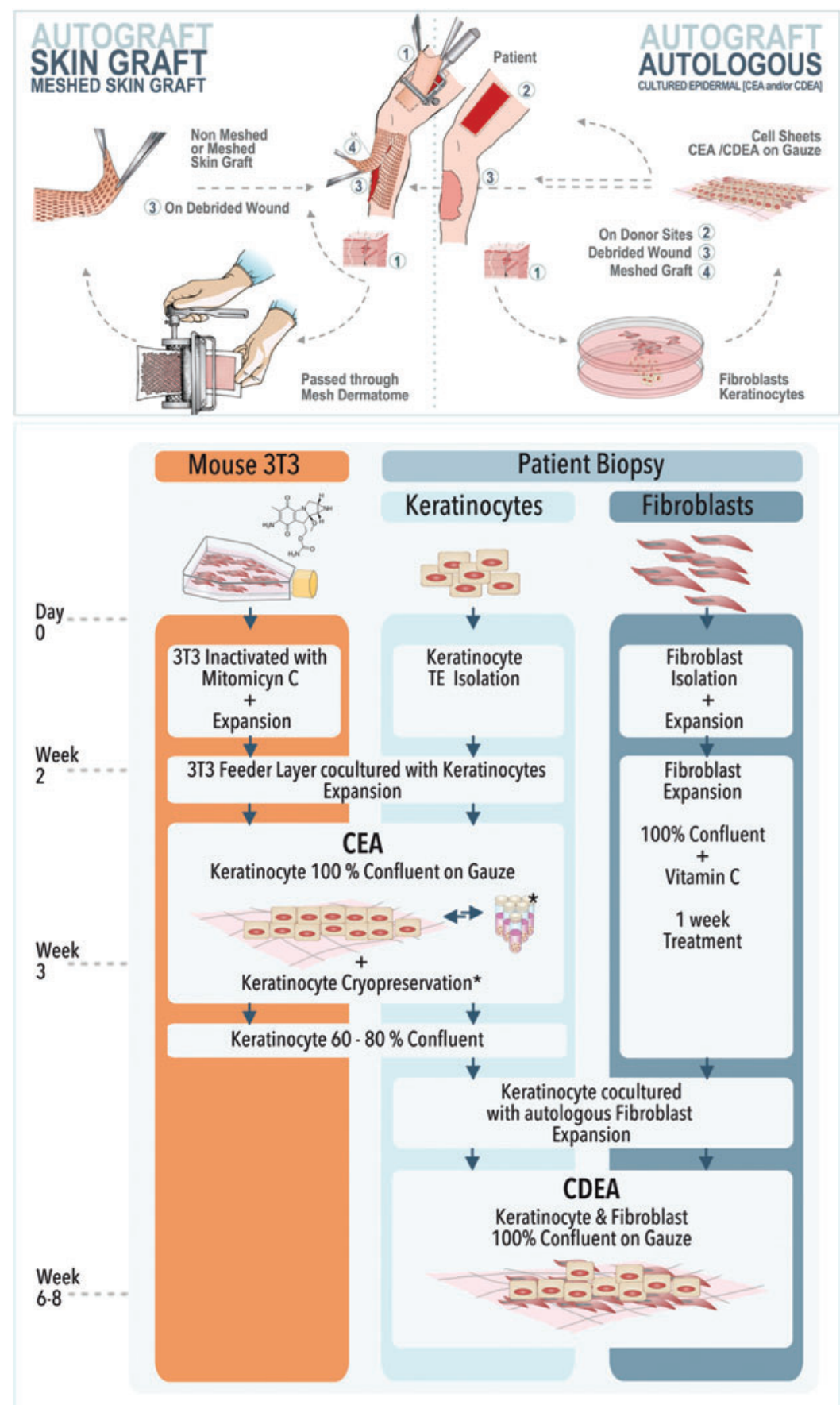

Figure 1. Upper panel: Schematic illustration of split-thickness skin autografting and cell-based autografting; lower panel: Fabrication process of CEA and CDEA. CDEA, cultured dermal-epidermal autograft; CEA, cultured epithelial autografting. Color images are available online.

present a brief overview of the different types of temporary skin substitutes.

\section{Conventional skin substitutes: human cadaveric and xenogeneic}

Several temporary skin substitutes were used over time as first cover for partial- and full-thickness burn wounds, for donor sites and meshed autograft protection. They have included human am- nion (e.g., Mimedx or Osiris), ${ }^{14,15}$ xenografts $^{16}$ (e.g., E-Z Derm ${ }^{\circledR}$, Mölnlycke, Sweden), and human cadaver allografts, such as supplied by the Euro Skin Bank (Beverwijk, Netherland). Porcine xenografts are probably the most frequently used temporary skin replacement worldwide due to easy accessibility and storage,${ }^{17}$ but also because porcine skin is anatomically close to human skin. ${ }^{18}$ Skin substitutes are available in several 


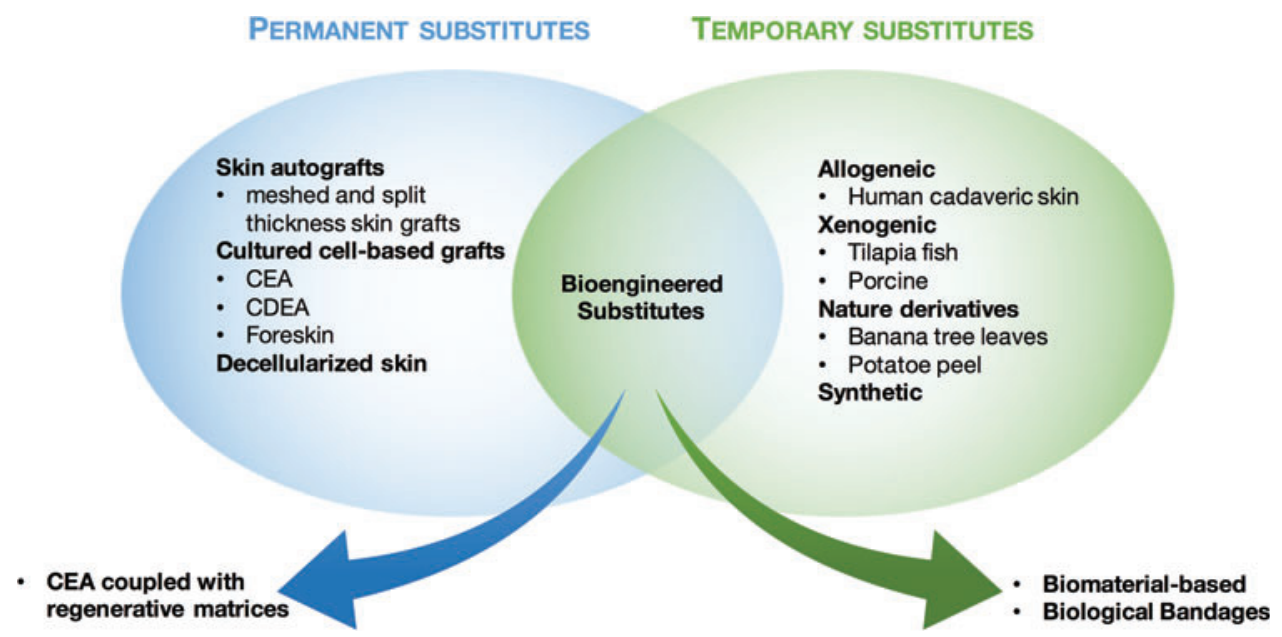

Figure 2. Skin substitutes classified into permanent and temporary substitutes. For both categories, products have evolved from conventional therapies to bioengineered products combining various cells and biomaterials as strategies to improve wound healing. Color images are available online.

forms: cryopreserved, in glycerol, lyophilized, and decellularized. ${ }^{16,19-21}$ It has been reported that storage in glycerol may be problematic as efficient removal of glycerol residues from the xenograft implies higher degradation rates of the substitute on patients. ${ }^{22}$

From our experience, cryopreserved allografts give better protection than glycerol-preserved allografts, probably because of a cryopreservation allowing dermal substitutes to retain the ability to release growth factors and biochemical cues promoting wound healing. ${ }^{23}$ It has been shown that an allogenic skin substitute becomes engrafted to the wound after 2-3 weeks, thus the alloepidermis is removed at surgery, leaving the dermal components as a viable bed for a CEA. ${ }^{24}$ In some cases, remaining allodermis parts even become re-epithelialized. ${ }^{25}$

The benefits of allografts and xenografts include immediate soothing upon application, minimized pain during dressing changes, and minimized protein and water loss, allowing the patient to resume activity without disturbing the healing process.

However, their main drawbacks are the rejectionrelated risks. Indeed, although the incorporated dermis is usually not rejected in a first period, allograft could trigger a chronic severe rejection reaction when the patient's immune system is restored. This can also appear clinically with the formation of unstable or hypertrophic scars. ${ }^{26}$ Furthermore, these temporary substitutes may enhance risk of disease transfer and their integration in the recipient site renders their ablation difficult and sometimes leaving painful eschars. ${ }^{8,27}$

\section{Synthetic substitutes}

Conventional skin substitutes are normally overlayered by low-adherent sterile paraffin gauze
(Jelonet from Smith \& Nephew Medical Ltd., England). On donor sites or when burn wounds are smaller, gauze or foams only are applied (synthetic dressings without allogenic/xenogeneic substitute) as standard of care. Currently $>3,000$ types of dressings are available on the market, offering a plethora of options to address all aspects of wound care. ${ }^{28} \mathrm{In}$ brief, these dressings encompass materials made of semipermeable silicone foams, hydrocolloids, hydrogels, and hydrofibers as reviewed by Borda et al. ${ }^{29}$

Although these dressings mainly protect the burn injury, they do not contribute to the recovery of dermal and epidermal tissues. Synthetic substitutes can also be dermal constructs made of nonbiological molecules and polymers not present in normal skin. Although these substitutes offer a stable and biodegradable environment for the regeneration of tissue with a more precisely controlled composition and properties, the use of nonbiological components can present biocompatible issues. ${ }^{30}$ Hence, there is still no superior synthetic substitute that can achieve complete healing, especially in case of chronic wounds such as venous leg ulcers, diabetic wounds, and pressure ulcers. ${ }^{31}$

Nevertheless, synthetic dressings or substitutes can be available in different formulations, thus several dressings are often functionalized with silver, iodine, or other antimicrobial agents to further prevent infection. ${ }^{32-34}$ However, current antimicrobials in use, most commonly silver, have the limitation to induce skin irritation and staining after prolonged treatment. ${ }^{35}$

\section{Bioengineered skin substitutes}

Contemporary technological advances in biomaterials have allowed to develop cell-based skin 
substitutes, such as Dermagraft ${ }^{\circledR}$ or Transcyte ${ }^{\circledR}$ from organogenesis (Canton), which are made, respectively, of polyglycolic acid/polylactic acid scaffolds and collagen-coated nylon mesh, both seeded with allogeneic foreskin fibroblasts secreting growth factors to aid wound healing. ${ }^{36}$

Other cell-free yet bioactive dressings have been developed such as Biobrane ${ }^{\circledR}$ (Smith \& Nephew, London, England), which consist of a semipermeable silicone film partially imbedded nylon, functionalized with porcine collagen. Biobrane has the benefit of minimizing bacteria proliferation on wound surface by minimizing dead space, controlling water vapor loss (comparable with native skin), and allowing conformability to surface irregularities. The latter property subsequently helps joint movement and early ambulation due to its flexible mechanical properties. A prospective randomized clinical trial comparing both bioengineered products, Transcyte and Biobrane, indicated that the cell-based Transcyte product promotes a faster re-epithelialization, whereas requiring fewer dressing changes and autografting procedures. ${ }^{37}$

Nevertheless, biocompatibility still remains the major issue for most of the developed bioengineered products, as they contain either allogeneic or xenogeneic components with risks of inflammation or rejection. To cope with this problem, we have developed at our hospital biological bandages made of clinical-grade progenitor skin fibroblasts of fetal origin, delivered to the burn wound on a collagen matrix. ${ }^{38}$ These bandages positively impact wound healing processes, most probably by secreting growth factors, while inducing minimal inflammatory response due to their immune-privileged source. Thus, we were able to show with eight pediatric patients treated with biological bandages alone that they did not require further associated autologous grafting. ${ }^{38}$

Likewise, progenitor cells showed remarkable outcomes in the treatment of donor site wounds (Fig. 3) and chronic wounds with positive outcome. ${ }^{39}$ The progenitor cells are derived from a Federal Transplantation Program following the Swiss constitution for laws on research on human beings and transplantation medicine (art 119a Cst). Fetal tissue is considered as an organ donation from voluntary pregnancy interruption and can be obtained with specific strict regulation within transplantation law.

The benefits of bioactive substitutes may be multiple depending on the severity of the burn wounds. For superficial wound zones, they allow a more rapid healing thus minimizing scar formation, whereas for intermediate zones, it can help to reduce the edema and risks of thrombosis of the capillaries; for deep wounds the bioactive components prepare the wound bed, thus improving subsequent grafting.

\section{Alternative natural skin covers}

Interestingly, skin substitutes derived directly from nature have also been used as temporary
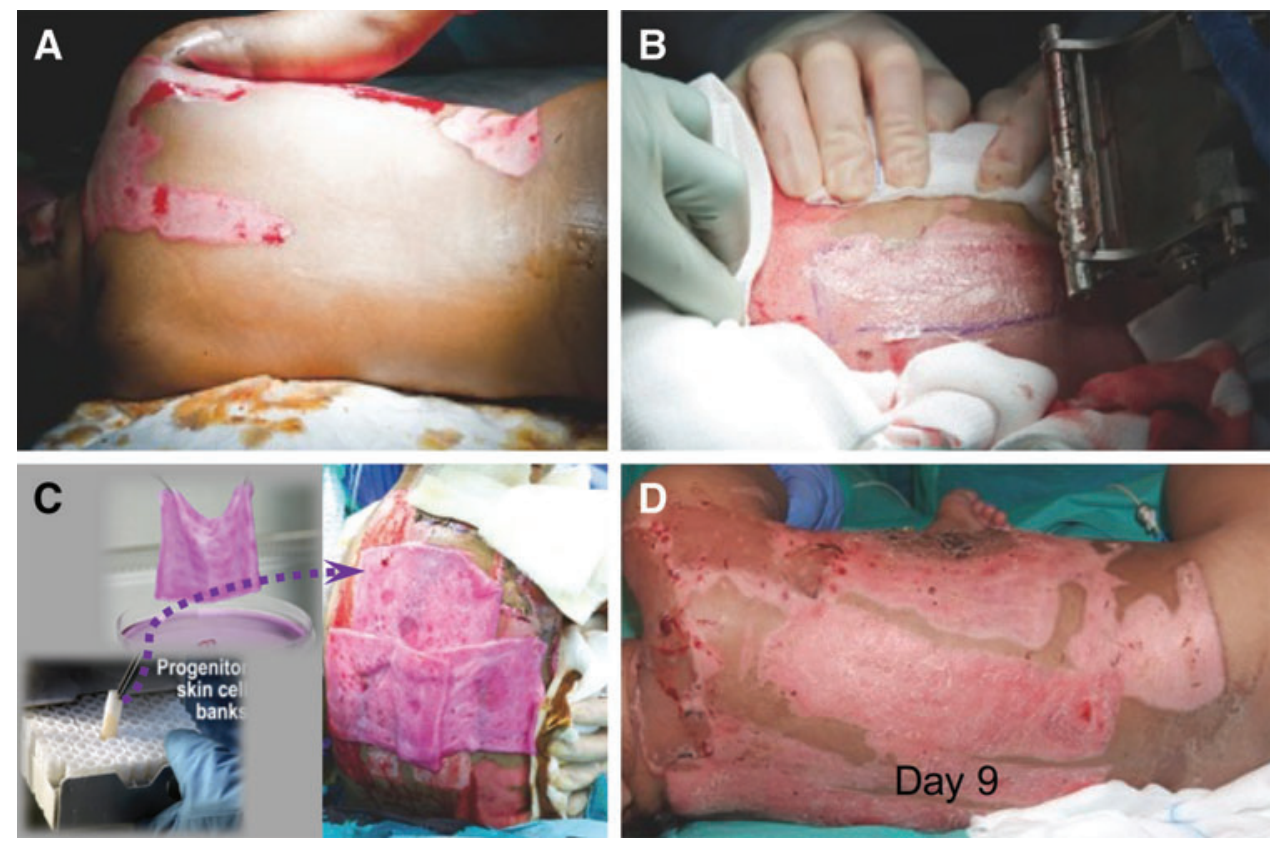

Figure 3. Use of biological bandages as cover for skin donor sites. (A) Back of pediatric patient with a deep burn at the right flank; (B) skin graft taken with dermatome on healthy sites of the back; (C) application of the biological bandages on the donor sites of the back; (D) re-epithelization of the skin donor sites after application of the biological bandages. Color images are available online. 
cover for burn patients, such as banana leaves, ${ }^{40}$ tilapia fish skin (e.g., Kerecis Omega3 fish skin) or potato peel. ${ }^{22}$ Such approaches are often in use in developing countries, where more sophisticated and more expensive wound cover are not easily available.

\section{BENCHMARKING OF FIRST COVERS}

In this section, we aim to give comparisons from our own experience between the different types of temporary substitutes used as first covers for burn wounds, thus particular focus will be given to cadaveric and porcine substitutes and the biological bandages, the three types of temporary covers employed at the burn unit of our institution (Fig. 4).

From a purely practical point of view, the advantage of the biological bandages over the cadaveric and porcine substitute resides is the fact that it can be easily removed from patients without being painful and leaving necrotic tissues (Fig. 5). Biological bandages, on the contrary to the allogenic substitute, do not require staples to attach to the patient, as they can be simply overlaid with Jelonet followed by a Multivac compress, as a standard of care.

Regarding the costs, the price of porcine cover (E-Z Derm of $8 \times 120 \mathrm{~cm}$ surface) is 321 Swiss Francs $(\mathrm{CHF})$, whereas a biological bandage $(9 \times 12 \mathrm{~cm})$ costs $26 \mathrm{CHF}$. Consequently, to cover $1 \mathrm{~m}^{2}$ of burn surface would cost about 3,340 $\mathrm{CHF}$ for porcine cover and 2,550 CHF for a biological bandage, which is $25 \%$ less expensive. The most expensive first cover remains human cadaveric skin substitute as it costs $\sim 12,000 \mathrm{CHF}$ for the same surface of $1 \mathrm{~m}^{2}$ (from Skin Euro Bank, Netherlands).

If we put into perspective these numbers with the costs of common dressings used in the standard of care of burn wounds, such as Aquacel (ConvaTec, United Kingdom) or a Jelonet paraffin Tulle Gras dressing, for the same surface a cadaveric cover is one order of magnitude more expensive than Aquacel, and two orders of magnitude more than Jelonet. Nevertheless, these numbers have to be normalized over time as well, since a biological bandage or conventional dressings can be changed several times per week, whereas conventional covers can remain several weeks without being changed.

The benefit of using such high-cost covers should then be weighted by the impact they might have on reducing hospitalization times, knowing that patient care at the Lausanne University Hospital (CHUV) Burn Unit costs 4,000 CHF per day. The main advantage of biological bandages over allogenic and xenogeneic covers, respectively, remains in the fact that it could actively stimulate wound healing. Indeed, as already mentioned, a biological bandage may induce faster healing minimizing thus scar formation, whereas for intermediate zones it can help to reduce the edema and risks of thrombosis in capillaries, and for deep zones it can prepare the wound bed thus improving subsequent grafting.

These advantages can positively impact wound healing that, in turn, may reduce hospitalization times and global costs, besides the fact that biological

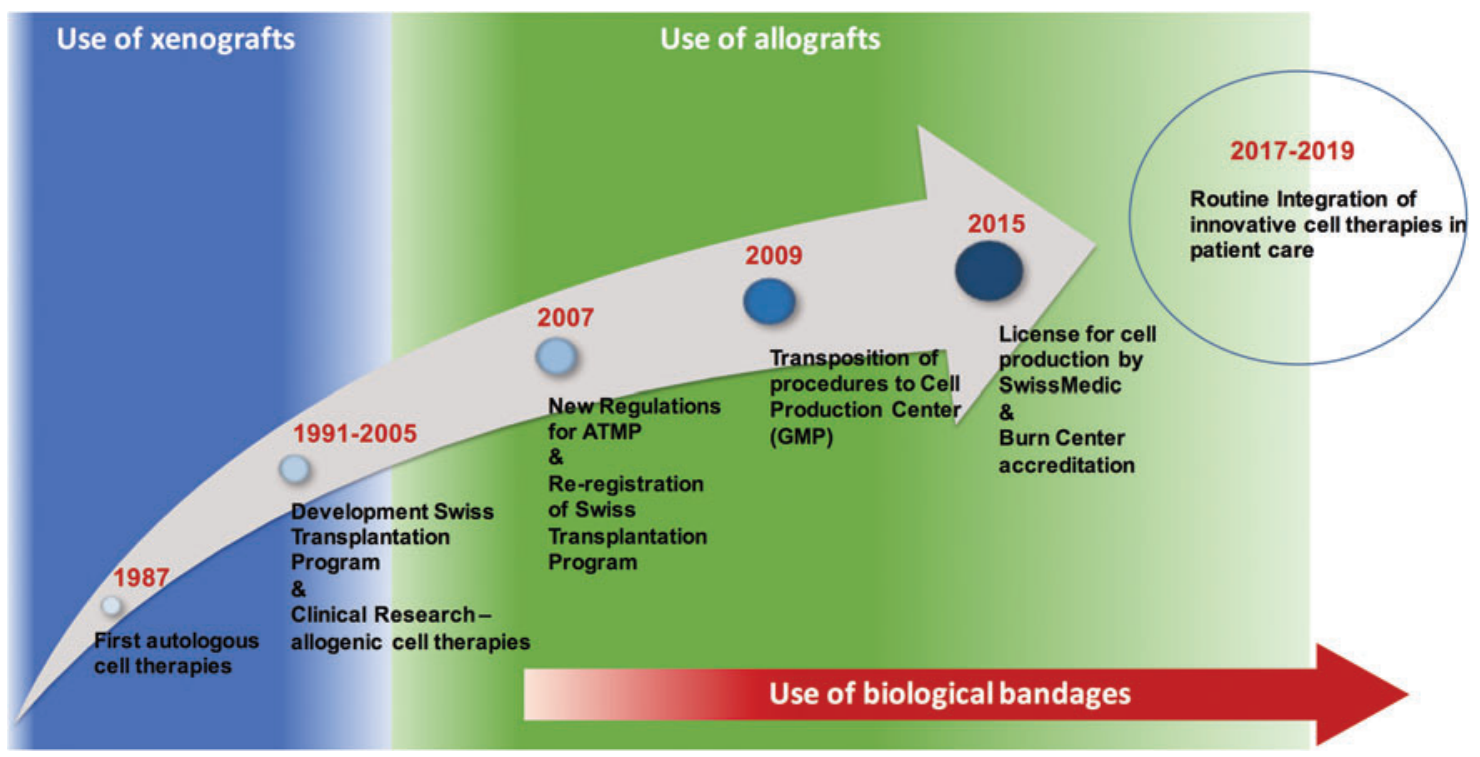

Figure 4. Timeline of evolution of the first covers used at CHUV burn unit and the implementation of cell therapies. Color images are available online. 

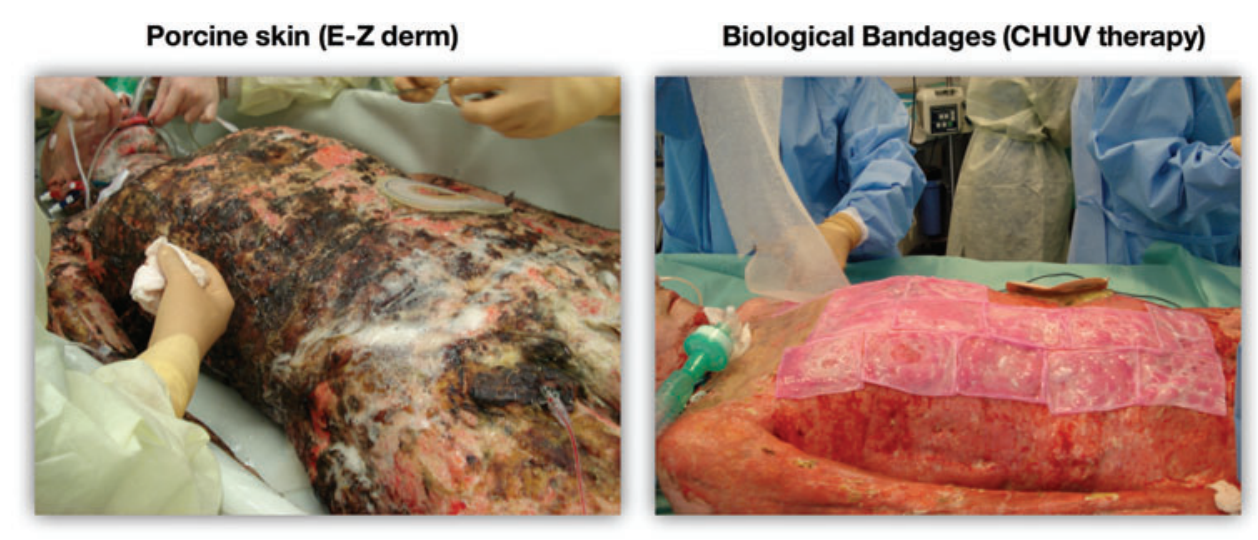

Figure 5. Photographs of a burn patient treated in a first time with E-Z Derm ${ }^{\circledR}$ as first cover (left pane), and with biological bandages (right pane/). Biological bandages are applied the same day after E-Z Derm removal and debridement; they demonstrate superior outcomes in comparison with porcine substitute, as shown by the lack of necrotic tissue, the absence of the staples and ease of handling. Color images are available online.

bandages can readily be prepared within $24 \mathrm{~h}$ from an off-the-shelf good manufacturing practice (GMP) cell bank, circumventing storage concerns.

Figure $6 \mathrm{~A}$ shows the constant increase in the number of biological bandages used at the Lausanne University Hospital per year between 2013 and 2017 , whereas the number of patients admitted to the ICU burn unit per year remains constant, which illustrated the implementation of biological bandages in clinical routine in burn care, especially for adult patients. This observation can also be illustrated by the increase of mean number of bandages used per patient over the years for a mean total body surface area (TBSA) per patient that also remains constant over time (Fig. 6B).

Interestingly, for the same year range, the indicator of care duration normalized by burned surface decreased from 0.6 days/TBSA in 2014 to 0.2 days/ TBSA in 2017 (for adults), thus suggesting an improvement in the quality of burn patient care, to which the use of biological bandages may have contributed, as all other aspects of burn care had been standardized previously, except for small improvements in nutrition intake, which was adjusted with supplement intake to cope with the exudative loss of trace elements within the first week. ${ }^{41}$

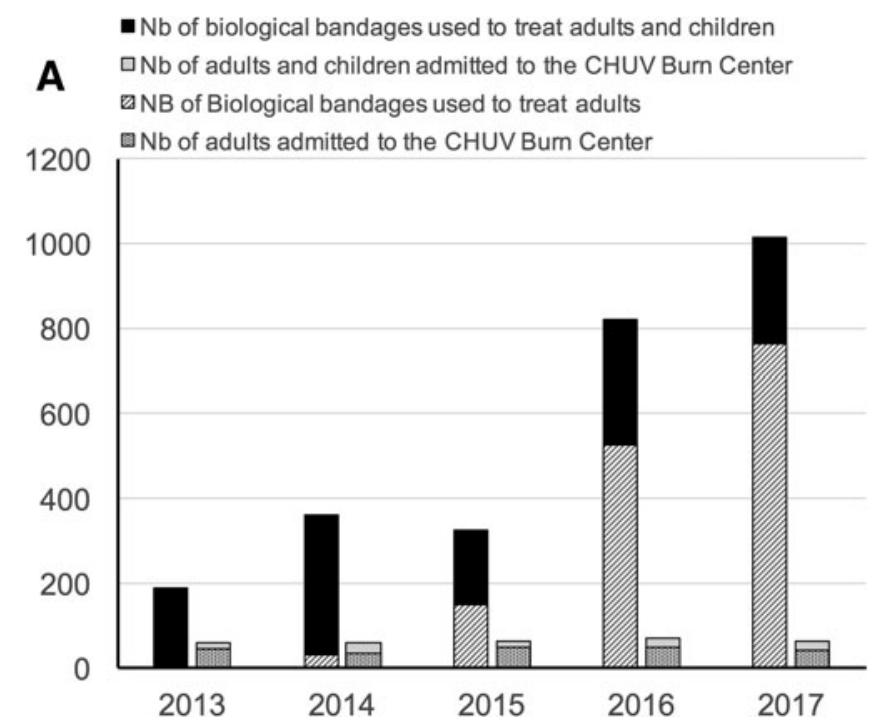

\section{B Mean TBSA/patient $\square$ Mean Nb of bandages/patient}

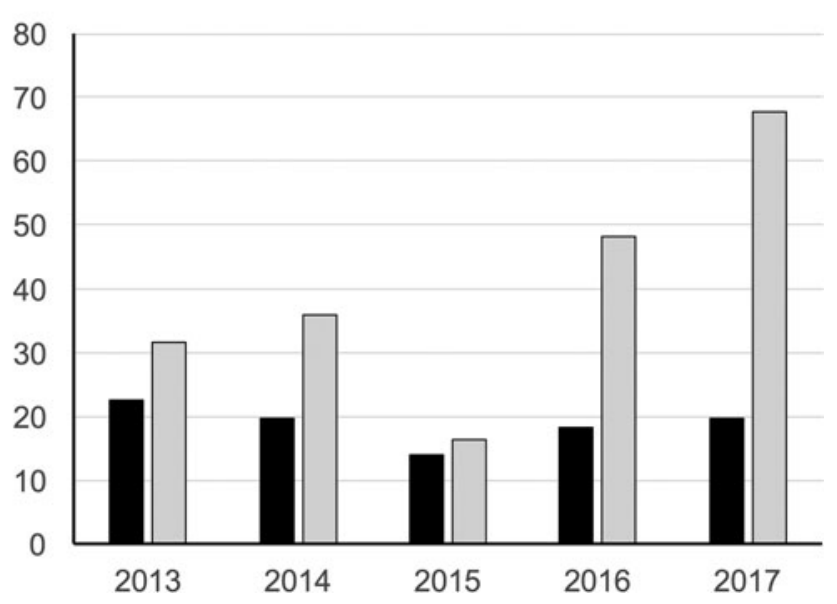

Figure 6. (A) Steady increase over time of production and use of biological bandages at the burn units of the Lausanne University Hospital, while number of patients admitted to the burn center has remained constant. We can see that this increase of use of biological bandages is mainly due to the introduction of this therapy as first cover for the adult patients. (B) Increase of the mean number of bandages used per patient, with a constant TBSA over time, which is relevant to the fact that this therapy has been more used in clinical routine and not because of higher burn surface. TBSA, total body surface area. 
Table 1. Benchmarking of the biological bandages and conventional allografts used as first covers

\begin{tabular}{lccc}
\hline & $\begin{array}{c}\text { Biological } \\
\text { bandage } \\
\text { (CHUV therapy) }\end{array}$ & $\begin{array}{c}\text { Porcine } \\
\left(\text { E-Z Derm }{ }^{\circledR}\right)\end{array}$ & $\begin{array}{c}\text { Cadaveric } \\
\text { (Euro Skin Bank) }\end{array}$ \\
\hline Resist infection & $+/-$ & + & + \\
High absorbency potential & ++ & - & - \\
Able to prevent water loss & + & + & + \\
Nonadhesive to ease removal & ++ & - & + \\
$\quad$ (produce less pain) & + & + & + \\
Withstand shear forces & + & - & + \\
Cost-effective & +- & + & + \\
Widely available & + & - & + \\
Long shelf life & ++ & + & + \\
Lack of antigenicity & ++ & & + \\
Conform to irregular & + & & + \\
$\quad$ wound surfaces & & & + \\
\hline
\end{tabular}

Table 1 summaries the assets that an "ideal" skin cover could have. ${ }^{22,42}$

Finally, a consideration that might affect the choice of a therapy or another is the use of certain biological material that might be related to religious and/or ethical issues (for instance porcine products for Muslim patients), which requires the informed consent of the patient.

\section{PERSPECTIVES ON FUTURE THERAPIES}

Despite early excision, immediate wound covering, and topical treatments, the major problematic remaining in burn patient care is the frequency increase of persistent wound infections due to hard-to-treat multidrug resistant bacteria, often Pseudomonas aeruginosa. ${ }^{43}$ This is especially true for large burn areas $>40-50 \%$. In an effort to cope with burn wound infections, we are currently developing a next generation of biological bandages, coupling antimicrobial dendrimers with progenitor cells, thus allowing a dual effect of controlled infection and enhanced wound healing. ${ }^{44}$

In the same perspective to improve wound healing, research endeavors are made to genetically edit keratinocytes to engineer skin equivalents, expressing factors that promote healing. ${ }^{16}$ Likewise, studies have shown that mesenchymal stem cells ${ }^{45}$ and adipose-derived stem cells ${ }^{46}$ can promote wound healing both by direct transfer onto wounds by delivery embedded in scaffolds, as these cells can secrete factors that attenuate inflammation and stimulate angiogenesis, thereby leading to faster wound closure. ${ }^{47}$

Although cell-based approaches show significant performance in wound healing, offering promising perspectives, they encounter some limitations due to regulatory hurdles. Indeed, since 2007 new EU regulations consider the application and practice of cell therapies such as "Advanced Therapy Medicinal Products" (ATMPs), imposing to cell therapies the same industry-destined GMPs as for pharmaceutical products. The aim of these regulations is to ensure the safety of these products to a maximum extent. However, these regulations imply considerable delays in research, development, and the production of cell therapies, which would not be of benefit to the patients, without mentioning the increase in costs.

We advocate for "Hospital Exemption," which would provide a more suitable pathway to customize safe product/therapy for access to industrialized patient care for burn victims. ${ }^{48}$ In this respect, revisions on the Therapeutic Products Act (Stage 2) have come into force, from January 2019, in Switzerland. These revisions would simplify and facilitate access to medicinal products for patients and improve conditions for biomedical research. In particular, it will entail a 2-year authorization for the "compassionate use" of a cell-based therapy provided that a clinical study has been approved in Switzerland before the use request.

Other European countries, for instance Belgium, have also applied the same kind of directives for the compassionate use, especially for unproven interventions such as the use of bacteriophages in the treatment of persistent recalcitrant infections, based on article 37 of the Declaration of Helsinki, which stipulates.

"In the treatment of an individual patient, where proven interventions do not exist or other known interventions have been ineffective, the physician, after seeking expert advice, with informed consent from the patient or a legally authorized representative, may use an unproven intervention if in the physician's judgement it offers hope of saving life, reestablishing health or alleviating suffering. This intervention should subsequently be made the object of research, designed to evaluate its safety and efficacy. In all cases, new information must be recorded and, where appropriate, made publicly available."

Therefore, we are now seeing that our policymakers are working hand-in-hand with those confronted to help patients in the best manner possible.

\section{CONCLUSIONS}

In conclusion, temporary dermal substitutes used as first cover for burn wounds can play a major role in skin regeneration as they allow a rapid covering of the wound and thus can significantly reduce infection risk by providing a physical barrier to pathogens, but they can also have an effect on the surface to be grafted and the 
rate of secondary corrective surgeries; therefore, first covers can indirectly reduce hospitalization time and global costs.

Patient informed consent is also an important matter to take into consideration as the use of various biological products might incite religious and/or ethical issues, which have not been notably documented in the medical literature for skin substitutes.

Advances have been made in the development of dermal substitutes in the past years, and further improvements in clinical outcome can be expected principally with the approach of cell-based therapies; however, hospital exemptions are necessary to assure safety for patients without hindering advances in therapies.

\section{SUMMARY}

First covers can improve clinical outcomes in skin regeneration, by allowing a rapid wound covering which subsequently can reduce infection risk, rate of secondary corrective surgeries, and thus hospitalization time and global costs. Recent advances have shown significant improvement of cell-based first cover for burn patients, thus offering promising perspectives in wound healing; nevertheless, cell-based approaches encounter some limitations due to regulatory hurdles.

Further improvements in clinical outcome can be expected; however, hospital exemptions are necessary to assure safety for patients without hindering advances in therapies.

\section{ACKNOWLEDGMENTS AND FUNDING SOURCES}

Prof. Applegate acknowledges previous funding from Sandoz and S.A.N.T.E foundations. Current funding from CHUV Priority Project is also acknowledged. Dr. Philippe Abdel-Sayed acknowledges funding from the Marie Sklodowska-Curie Action. The authors thank Ms. Murielle Michetti and Ms. Corinne Scaletta for technical support with artwork.

\section{AUTHOR DISCLOSURE AND GHOSTWRITING}

Prof. Lee Ann Applegate is the cofounder and interim CEO of Elanix Biotechnologies. Prof.
Wassim Raffoul, Dr. Anthony de Buys Roessingh, and Dr. Nathalie Hirt-Burri are cofounders of Elanix Biotechnologies. The content of this article was expressly written by the authors listed and no ghostwriters were used.

\section{ABOUT THE AUTHORS}

Dr. Philippe Abdel-Sayed, $\mathbf{P h D}$, is a research associate at the Regenerative Therapy Unit at University Hospital of Lausanne, Switzerland. His research focuses on the development of novel generation of antimicrobial bandages to improve wound healing. Dr. Nathalie Hirt-Burri, PhD, is a research associate at the Regenerative Therapy Unit at University Hospital of Lausanne, Switzerland. Her research focuses on further development of cell-based therapies for burns. Dr. Anthony de Buys Roessingh, MD/PhD, is a senior surgeon at the Service of Pediatric Surgery the University Hospital of Lausanne, Switzerland; he is among the pioneers who have implemented the use of biological bandages as standard of care in the treatment of burned children. Prof. Wassim Raffoul, MD, is the head of the Service of Plastic, Reconstructive and Hand Surgery (CPR) at the University Hospital of Lausanne, Switzerland. CPR is responsible for implementing the cell therapies at the CHUV Burn Unit. Prof. Lee Ann Applegate, PhD, is an associate professor in the Department Musculoskeletal Medicine at the University Hospital of Lausanne, Switzerland. Research in her laboratory focuses on innovative cell therapy strategies for the treatment of burn wounds, including the development of the biological bandages. 


\section{REFERENCES}

1. Madison KC. Barrier function of the skin: "la raison d'etre" of the epidermis. J Invest Dermatol 2003;121:231-241.

2. Berardesca E, Maibach H. Ethnic skin: overview of structure and function. J Am Acad Dermatol 2003; 48:S139-S142.

3. Wojtowicz AM, Oliveira S, Carlson MW, Zawadzka A, Rousseau CF, Baksh D. The importance of both fibroblasts and keratinocytes in a bilayered living cellular construct used in wound healing. Wound Repair Regen 2014;22:246-255.

4. DiPietro LA. Angiogenesis and wound repair: when enough is enough. J Leukoc Biol 2016;100:979-984.

5. Sen CK, Gordillo GM, Roy S, et al. Human skin wounds: a major and snowballing threat to public health and the economy. Wound Repair Regen 2009;17:763-771.

6. Simman R, Phavixay L. Split-thickness skin grafts remain the gold standard for the closure of large acute and chronic wounds. J Am Col Certif Wound Spec 2011;3:55-59.

7. Rheinwald JG, Green H. Serial cultivation of strains of human epidermal keratinocytes: the formation of keratinizing colonies from single cells. Cell 1975;6:331-343.

8. Koch N, Erba P, Benathan M, Raffoul W. New developments in skin reconstruction - cell cultures and skin substitutes plus review of the literature [in French]. Ann Burns Fire Disasters 2010;23:131-136.

9. Saffle JR. Closure of the excised burn wound: temporary skin substitutes. Clin Plast Surg 2009;36:627-641.

10. Bloemsma GC, Dokter J, Boxma H, Oen IM. Mortality and causes of death in a burn centre. Burns 2008;34:1103-1107.

11. Cumming J, Purdue GF, Hunt JL, O'Keefe GE. Objective estimates of the incidence and consequences of multiple organ dysfunction and sepsis after burn trauma. J Trauma 2001;50:510-515.

12. Peck MD. Epidemiology of burns throughout the world. Part I: distribution and risk factors. Burns 2011;37:1087-1100

13. Fournier A, Eggimann $P$, Pagani $\mathrm{JL}$, et al. Impact of the introduction of real-time therapeutic drug monitoring on empirical doses of carbapenems in critically ill burn patients. Burns 2015;41:956968.

14. Robson MC, Krizek TJ. The effect of human amniotic membranes on the bacteria population of infected rat burns. Ann Surg 1973;177:144-149.

15. Robson MC, Krizek TJ, Koss N, Samburg JL. Amniotic membranes as a temporary wound dressing. Surg Gynecol Obstet 1973;136:904-906.

16. Sun BK, Siprashvili Z, Khavari PA. Advances in skin grafting and treatment of cutaneous wounds. Science 2014;346:941-945.

17. Hermans MH. Porcine xenografts vs. (cryopreserved) allografts in the management of partial thickness burns: is there a clinical difference? Burns 2014;40:408-415.
18. Sullivan TP, Eaglstein WH, Davis SC, Mertz P. The pig as a model for human wound healing. Wound Repair Regen 2001;9:66-76.

19. Maral T, Borman H, Arslan H, Demirhan B, Akinbingol G, Haberal M. Effectiveness of human amnion preserved long-term in glycerol as a temporary biological dressing. Burns 1999;25:625-635.

20. Ravishanker R, Bath AS, Roy R. "Amnion Bank" - the use of long term glycerol preserved amniotic membranes in the management of superficial and superficial partial thickness burns. Burns 2003;29:369-374.

21. Shevchenko RV, James SL, James SE. A review of tissue-engineered skin bioconstructs available for skin reconstruction. J R Soc Interface 2010;7:229-258.

22. Halim AS, Khoo TL, Mohd Yussof SJ. Biologic and synthetic skin substitutes: an overview. Indian $\mathrm{J}$ Plast Surg 2010;43:S23-S28.

23. Kubo K, Kuroyanagi Y. The possibility of long-term cryopreservation of cultured dermal substitute. Artif Organs 2005;29:800-805.

24. Hickerson WL, Compton C, Fletchall S, Smith LR. Cultured epidermal autografts and allodermis combination for permanent burn wound coverage. Burns 1994;20(Suppl 1):S52-S55; discussion S55-S56.

25. Hettich R, Ghofrani A, Hafemann B. The immunogenicity of glycerol-preserved donor skin. Burns 1994;20(Suppl 1):S71-S75; discussion S75-S76.

26. Shores JT, Gabriel A, Gupta S. Skin substitutes and alternatives: a review. Adv Skin Wound Care 2007;20:493-508; quiz 509-510.

27. May SR. The effects of biological wound dressings on the healing process. Clin Mater 1991;8:243-249.

28. Dhivya S, Padma W, Santhini E. Wound dressingsa review. Biomedicine (Taipei) 2015;5:22.

29. Borda LJ, Macquhae FE, Kirsner RS. Wound dressings: a comprehensive review. Curr Dermatol Rep 2016:5:287-297.

30. van der Veen VC, van der Wal MB, van Leeuwen MC, Ulrich MM, Middelkoop E. Biological background of dermal substitutes. Burns 2010;36:305-321.

31. Derakhshandeh H, Kashaf SS, Aghabaglou F, Ghanavati IO, Tamayol A. Smart bandages: the future of wound care. Trends Biotechnol 2018;36:1259-1274.

32. Fursatz $M$, Skog M, Sivler $P$, et al. Functionalization of bacterial cellulose wound dressings with the antimicrobial peptide epsilon-poly-L-Lysine. Biomed Mater 2018;13:025014.

33. Gueltzow M, Khalilpour P, Kolbe K, Zoellner $Y$. Budget impact of antimicrobial wound dressings in the treatment of venous leg ulcers in the German outpatient care sector: a budget impact analysis. J Mark Access Health Policy 2018;6:1527654.

34. Hu S, Cai X, Ou X, et al. Preparation of biocompatible wound dressings with long-term antimicrobial activity through covalent bonding of antibiotic agents to natural polymers. Int J Biol Macromol 2019;123:1320-1330.

35. Group A, Lea A. Contact dermatitis with a highlight on silver: a review. Wounds 2010;22:311-315.
36. Varkey M, Ding J, Tredget EE. Advances in skin substitutes-potential of tissue engineered skin for facilitating anti-fibrotic healing. J Funct Biomater 2015;6:547-563.

37. Kumar RJ, Kimble RM, Boots R, Pegg SP. Treatment of partial-thickness burns: a prospective, randomized trial using Transcyte. ANZ J Surg 2004;74:622-626

38. Hohlfeld J, de Buys Roessingh A, Hirt-Burri N, et al. Tissue engineered fetal skin constructs for paediatric burns. Lancet 2005;366:840-842.

39. Ramelet AA, Hirt-Burri N, Raffoul W, et al. Chronic wound healing by fetal cell therapy may be explained by differential gene profiling observed in fetal versus old skin cells. Exp Gerontol 2009;44:208-218.

40. Gore MA, Akolekar D. Evaluation of banana leaf dressing for partial thickness burn wounds. Burns 2003;29:487-492

41. Jafari P, Thomas A, Haselbach D, et al. Trace element intakes should be revisited in burn nutrition protocols: a cohort study. Clin Nutr 2018;37:958-964.

42. Selig HF, Lumenta DB, Giretzlehner M, Jeschke MG, Upton D, Kamolz LP. The properties of an "ideal" burn wound dressing — what do we need in daily clinical practice? Results of a worldwide online survey among burn care specialists. Burns 2012;38:960-966.

43. Appelgren P, Bjornhagen V, Bragderyd K, Jonsson $\mathrm{CE}$, Ransjo U. A prospective study of infections in burn patients. Burns 2002;28:39-46.

44. Abdel-Sayed P, Kaeppeli A, Siriwardena T, et al. Anti-microbial dendrimers against multidrugresistant $P$. aeruginosa enhance the angiogenic effect of biological burn-wound bandages. Sci Rep 2016;6:22020

45. Jackson WM, Nesti LJ, Tuan RS. Concise review: clinical translation of wound healing therapies based on mesenchymal stem cells. Stem Cells Transl Med 2012;1:44-50

46. Mizuno H, Tobita M, Uysal AC. Concise review: adipose-derived stem cells as a novel tool for future regenerative medicine. Stem Cells 2012;30: 804-810.

47. Maranda EL, Rodriguez-Menocal L, Badiavas EV Role of mesenchymal stem cells in dermal repair in burns and diabetic wounds. Curr Stem Cell Res Ther 2017:12:61-70.

48. Dimitropoulos $G$, Jafari $P$, de Buys Roessingh $A$, Hirt-Burri N, Raffoul W, Applegate LA. Burn patient care lost in good manufacturing practices? Ann Burns Fire Disasters 2016;29:111-115.

\section{Abbreviations and Acronyms}

ATMP $=$ Advanced Therapy Medicinal Product CDEA $=$ cultured dermal-epidermal autograft

CEA $=$ cultured epithelial autografting

$\mathrm{CHF}=$ Swiss Francs

CHUV $=$ Lausanne University Hospital

GMP $=$ good manufacturing practice

TBSA $=$ total body surface area 